Supporting information

\title{
2D Black Phosphorus-Based Cytomembrane Mimics with Stimuli- Responsive Antibacterial Action Inspired by Endotoxin-Associated Toxic Behavior
}

Wenxin Liu, ${ }^{a, b \neq}$ Yingnan Zhu, ${ }^{c \neq}$ Qianqian Liu, ${ }^{a, b}$ Duo Wang, ${ }^{a, b}$ Zhaofan Tao, ${ }^{a, b}$ Haile Zhao, ${ }^{a, b}$ Haixia Wu, ${ }^{a, b}$ Lei Zhang, ${ }^{d, *}$ and Alideertu Dong ${ }^{a, b, *}$

${ }^{a}$ College of Chemistry and Chemical Engineering, Inner Mongolia University, Hohhot 010021, People's Republic of China ${ }^{b}$ Engineering Research Center of Dairy Quality and Safety Control Technology, Ministry of Education, Inner Mongolia University, Hohhot 010021, People's Republic of China 'School of Pharmaceutical Sciences, Center for Drug Safety Evaluation and Research, Zhengzhou University, Zhengzhou 450001, People's Republic of China ${ }^{\mathrm{d} D e p a r t m e n t}$ of Biochemical Engineering, School of Chemical Engineering and Technology, Frontier Science Center for Synthetic Biology and Key Laboratory of Systems Bioengineering (MOE), Collaborative Innovation Center of Chemical Science and Engineering (Tianjin), Tianjin University, Tianjin 300350, People's Republic of China

*E-mail: lei_zhang@tju.edu.cn (Lei Zhang); dongali@imu.edu.cn (Alideertu Dong). 


\section{Experimental Section}

\section{Materials}

Bulk black phosphorus (BP) was obtained from Nanjing XFNANO Materials Tech Co., Ltd. Sodium hydroxide $(\mathrm{NaOH})$ was obtained from Tianjing Fengchuan Chemical Reagent Technologies CO., Ltd. N-methyl-2-pyrrolidone (NMP), 1-vinylimidazole, 1bromopentane and cucurbituril $(\mathrm{CB}[7])$ were purchased from Shanghai Macklin Biochemical Co., Ltd. The culture medium components, containing yeast extract powder (Beijing Aoboxing Biotech Co., Ltd.), tryptone (Oxoid Co., Ltd.), and agar (Biosharp Co., Ltd.) were biological-reagent grade. Ultrapure water was used in all experiments and was supplied by a Millipore system (Millipore Inc.). Other chemicals were analytical grade and were utilized without purification.

\section{Preparation of black phosphorus (BP)}

BP nanosheets (BP) were synthesized by basic solvent exfoliation from bulk BP. Typically, $130 \mathrm{mg}$ of bulk BP crushed in advance was added to $150 \mathrm{~mL}$ of a saturated $\mathrm{NaOH} / \mathrm{NMP}$ solution. Then the above mixture was ultra-sonicated at $25 \mathrm{KHz}$ and $90 \%$

power using an ultrasonic homogenizer (JY92-IIN, Ningbo Scientz Biotechnology CO., Ltd) for $10 \mathrm{~h}$ in an ice bath. Then the dispersion was centrifuged at 1,000 rpm using a laboratory centrifuge for $30 \mathrm{~min}$ to remove the non-exfoliated BP, then the supernatant was centrifuged at 15,000 rpm for 30 min to obtain the ultrathin BP. All the precipitates were washed two times with anhydrous ethanol and ultrapure water, and the final products were obtained after vacuum freeze-drying treatment.

\section{Synthesis of PQVI}

Typically, 1-vinylimidazole $(20 \mathrm{mmol})$ and 1-bromopentane $(20 \mathrm{mmol})$ were added into $5 \mathrm{~mL}$ of cyclohexane. The homopolymer PQVI was obtained after the above mixture was stirred at $55{ }^{\circ} \mathrm{C}$ for $24 \mathrm{~h}$ under a $\mathrm{N}_{2}$ gas inlet. After the product was frozen overnight, $10 \mathrm{~mL}$ of tetrahydrofuran was added and stirred out. The final PQVI was achieved by vacuum drying the precipitated solids after filtration and washing.

\section{Synthesis of BP-PQVI}


BP-PQVI was combined through electrostatic interaction. Briefly, $10 \mathrm{mg}$ of BP and $100 \mathrm{mg}$ of PQVI were dispersed in $10 \mathrm{~mL}$ of ultrapure water with $\mathrm{pH}$ at 7 and then were ultrasonicated for $5 \mathrm{~h}$. Then the mixture were stirred for $12 \mathrm{~h}$ at room temperature and the dispersion was centrifuged by centrifuge. The collections were washed three times with ultrapure water and treated with vacuum freeze-drying.

\section{Characterization}

The morphology, size and surface state of the products were recorded on a Hitachi H8100 transmission electron microscope (TEM) at $8 \mathrm{kV}$ and a Shimadzu SSX-550 field emission scanning electron microscope $(\mathrm{SEM})$ at $15.0 \mathrm{kV}$. EDS elemental mapping of different elements were performed on a JEOL Jem-2100F high-resolution transmission electron microscope (HRTEM). XPS spectra were achieved with the ESCALAB 250Xi XPS system (Thermo Fisher Scientific) and monochromated Al-K radiation (1486.6 eV, $150 \mathrm{~W})$. FT-IR spectra were obtained by the Thermo Nicolet 6700 spectrometer using potassium bromide $(\mathrm{KBr})$ as the background. ${ }^{1} \mathrm{H}$ NMR spectra were recorded on a Bruker AVANCE III-500 NMR instrument using $\mathrm{D}^{2} \mathrm{O}$ as solvent. The morphology, surface state, and thickness were observed with a Bruker Dimension Icon atomic force microscope (AFM) in the tapping mode. Ultraviolet-visible (UV-vis) spectra were measured with a UV-vis spectrophotometer (U-3900). The Raman spectra were observed using a HORIBA Scientific LabRAM HR Evolution Raman spectrometer with an excitation laser wavelength of $633 \mathrm{~nm}$ at room temperature.

\section{Computer simulation methods}

The primitive cell structure of BP was download from crystal open database (COD ID:1010325), a super cell was constructed by $6 * 6 * 1$. The density theory functional (DFT) method was at B3LYP/6-311+G basis set level gaussian09d01 software package. After that coordinates and net charge were saved as mol2 type file, preparing for the adsorption simulation next step. First adsorption interactions were simulated with MS8.0 adsorption locator module by simulated annealing method and ultrafine level. All other parameters were set as default. 
Molecular dynamic simulation was finished with MS8.0 forcite module, adding the adsorbed molecule (or ion), then water molecules were added with the density $1 \mathrm{~g} / \mathrm{cm}^{3}$ and building the water solvent model. First stage was geometry optimization to elimination of unreasonable interatomic overlap. In the MD process UFF molecular force field and ultrafine quality was employed, Ewald method was selected for electrostatic interactions, and atom based method for van der waals force calculation and NVE ensemble system for the model. The initial velocity was random, the simulation time step was $1 \mathrm{fs}$ and the total simulation time is $5000 \mathrm{ps}(5 \mathrm{~ns})$. All other parameters set as default.

\section{Antibacterial assay}

The antibacterial activities of the products were determined by counting CFUs of the survivals. The active bacterial cells in the culture described above $\left(10^{8}-10^{9} \mathrm{CFU} \mathrm{mL} \mathrm{mL}^{-1}\right)$ were centrifuged and washed three times with normal saline. Subsequently, $100 \mu \mathrm{L}$ of the above bacterial suspension was mixed with $900 \mu \mathrm{L}$ of sample dispersions and shaken at room temperature for $3 \mathrm{~h}$. The mixture was then diluted and $1 \mathrm{~mL}$ of the as-diluted mixture was spread on LB agar plates and cultivated at $37^{\circ} \mathrm{C}$ for $12 \mathrm{~h}$. All tests were performed in triplicate, the surviving colonies on each LB agar plate were counted, and the corresponding antibacterial rates were calculated using the following equation (1):

Antibacterial rate $\%=(B-A) / B \times 100 \%$

where $A$ is the number of surviving colonies in the test sample and $B$ is the number of surviving colonies in the control.

\section{Minimum inhibitory concentration (MIC) assay}

Bacteria were cultured in Luria-Bertani (LB) medium for $12 \mathrm{~h}$ at $37{ }^{\circ} \mathrm{C}$ under shaking at $220 \mathrm{rpm}$. After centrifugation at $4000 \mathrm{rpm}$ for $5 \mathrm{~min}$, bacteria in the culture medium were collected and re-suspended in $\mathrm{LB}$ medium to $10^{5} \mathrm{CFU} \mathrm{mL}^{-1}$ as the working suspension. Samples were diluted to concentrations ranging from $4 \mu \mathrm{g} \mathrm{mL} \mathrm{mL}^{-1}$ to $2048 \mu \mathrm{g}$ $\mathrm{mL}^{-1}$ by a two-fold gradient dilution in a 96-well plate. After mixing equal volumes of bacterial cells suspension in each well, the 96-well plates were incubated at $37{ }^{\circ} \mathrm{C}$ for 12 $\mathrm{h}$ to collect $\mathrm{OD}$ values on a SpectraMax ${ }^{\circledR}$ M2 plate reader. LB medium was used as the 
blank, and bacteria in LB medium was used as the positive control. Measurements were performed in duplicates, and the experiments were repeated at least twice. The percentage of bacterial cells survival was calculated from the equation (2):

$$
\text { Cell growth } \%=\left(\mathrm{A}_{\text {sample }}-\mathrm{A}_{\text {blank }}\right) /\left(\mathrm{A}_{\text {control }}-\mathrm{A}_{\text {blank }}\right) \times 100 \%
$$

9. In vivo wound healing test

In vivo wound healing tests were conducted on mice from the Laboratory Animal Center of Inner Mongolia University. Prior to the surgery, the mice were anesthetized using an intraperitoneal injection of $10 \%$ chloral hydrate (an average of $0.06 \mathrm{~mL}$ per mouse). Wounds were created by performing a full-thickness incision with a diameter of $3 \mathrm{~mm}$ on the back of each mouse. The tests were carried out on three groups, including control group, BP-PQVI group, and BP-PQVI with $\mathrm{Mg}^{2+}$ group, and each group contained six mice. Then $100 \mu \mathrm{L}$ of E. coli $\left(10^{7} \mathrm{CFU} \mathrm{mL}^{-1}\right)$ was dropped on the wound surface and the control group that received without wound treatment, and was observed for six days. Six healthy mice selected as comparatives were monitored as well. The wound closures were measured every day and calculated using equation (3):

$$
\text { wound closure }(\%)=\left(1-A_{t} / A_{0}\right) \times 100 \%
$$

where $A_{0}$ is the initial wound area and $A_{t}$ is the wound area after a specific time interval.

When the mice were sacrificed 6 days after surgery, tissue and blood samples from around the wound were collected and homogenized in physiological saline. The diluted tissue solutions were incubated on an LB medium, and the survival colonies were counted after $24 \mathrm{~h}$. The tissue sections were stained with hematoxylin and eosin (H\&E) and observed by light microscopy. Then the eyeball bloods of the treated mice were analyzed to measure white blood cell (WBC) level and Gran level. These experiments were performed with the approval of the laboratory Animal Centre of the Inner Mongolia University.

\section{Statistical Analysis}

All data measurements and analyses are averages \pm standard deviation. For all comparisons, $\mathrm{p}=0.05$ is considered statistically significant. 
Figures

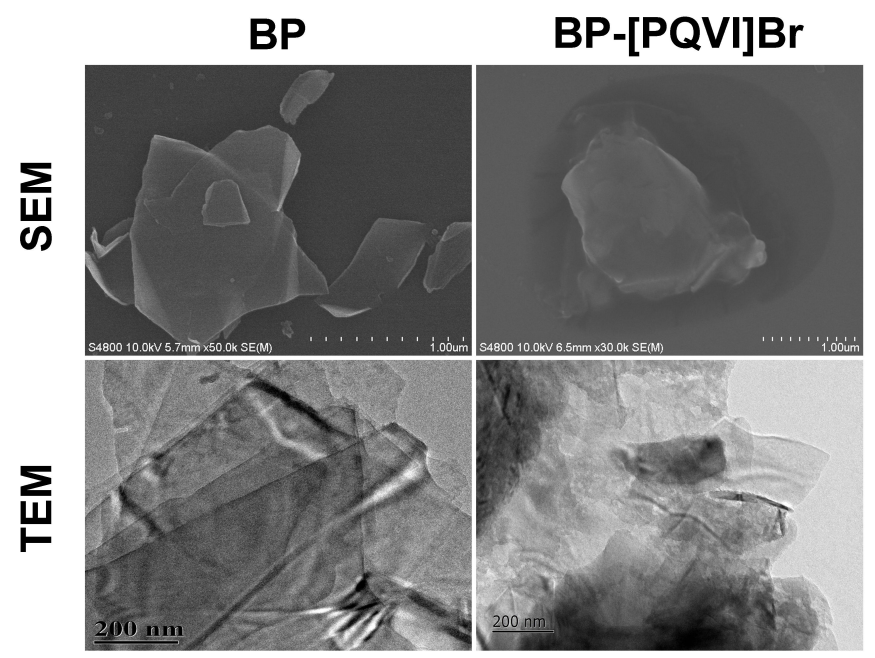

Figure S1. SEM and TEM images of BP and BP-PQVI. 


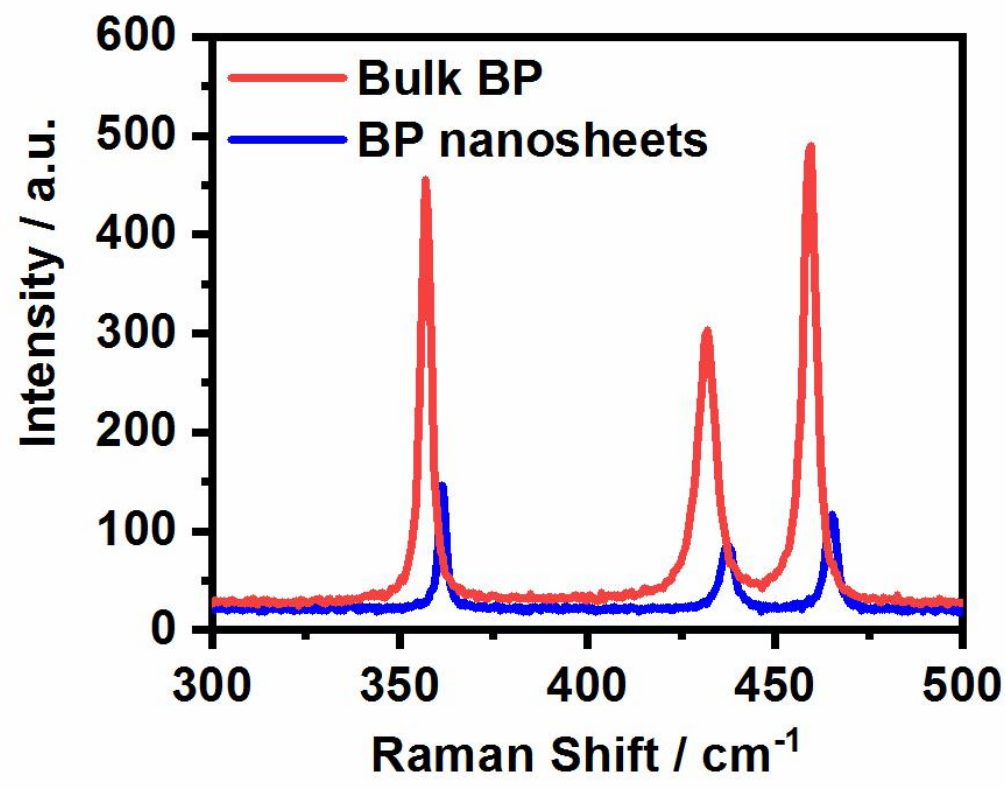

Figure S2. Raman spectra of bulk BP and BP nanosheets. 


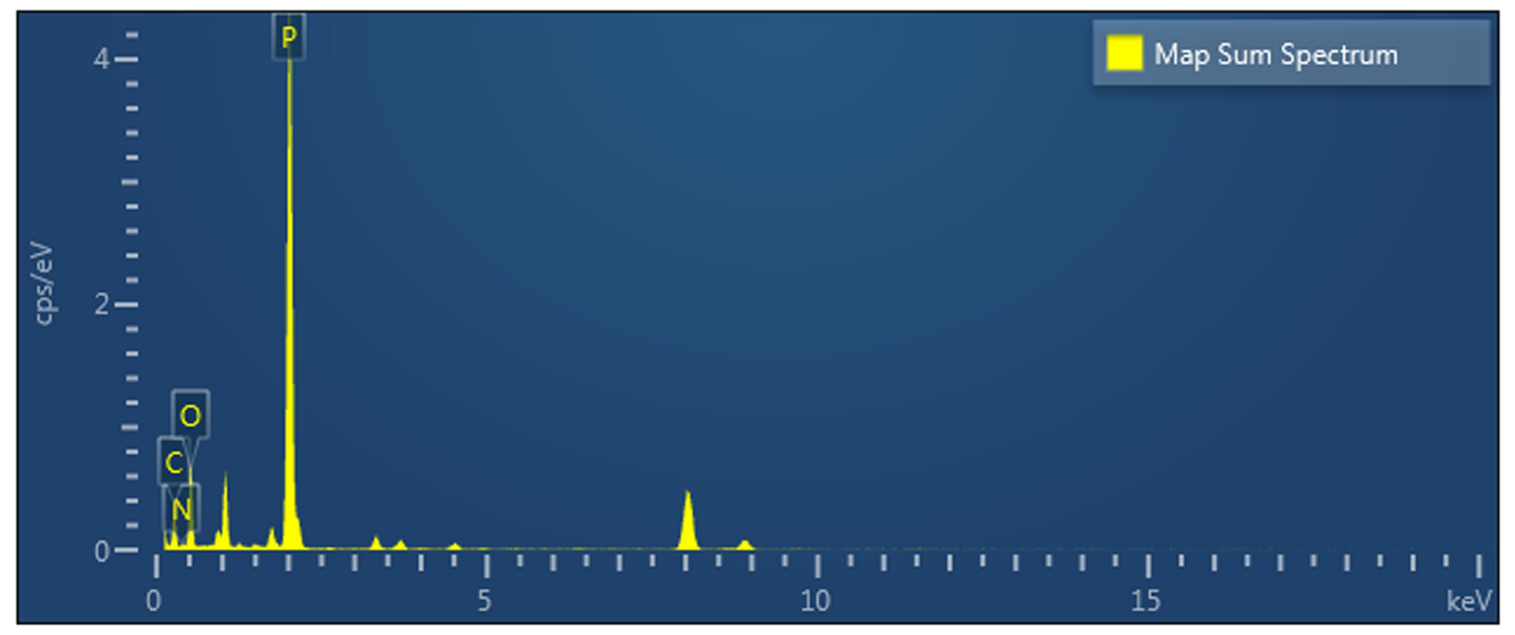

Figure S3. Dispersive X-ray spectroscopy (EDX) analysis of BP-PQVI. 

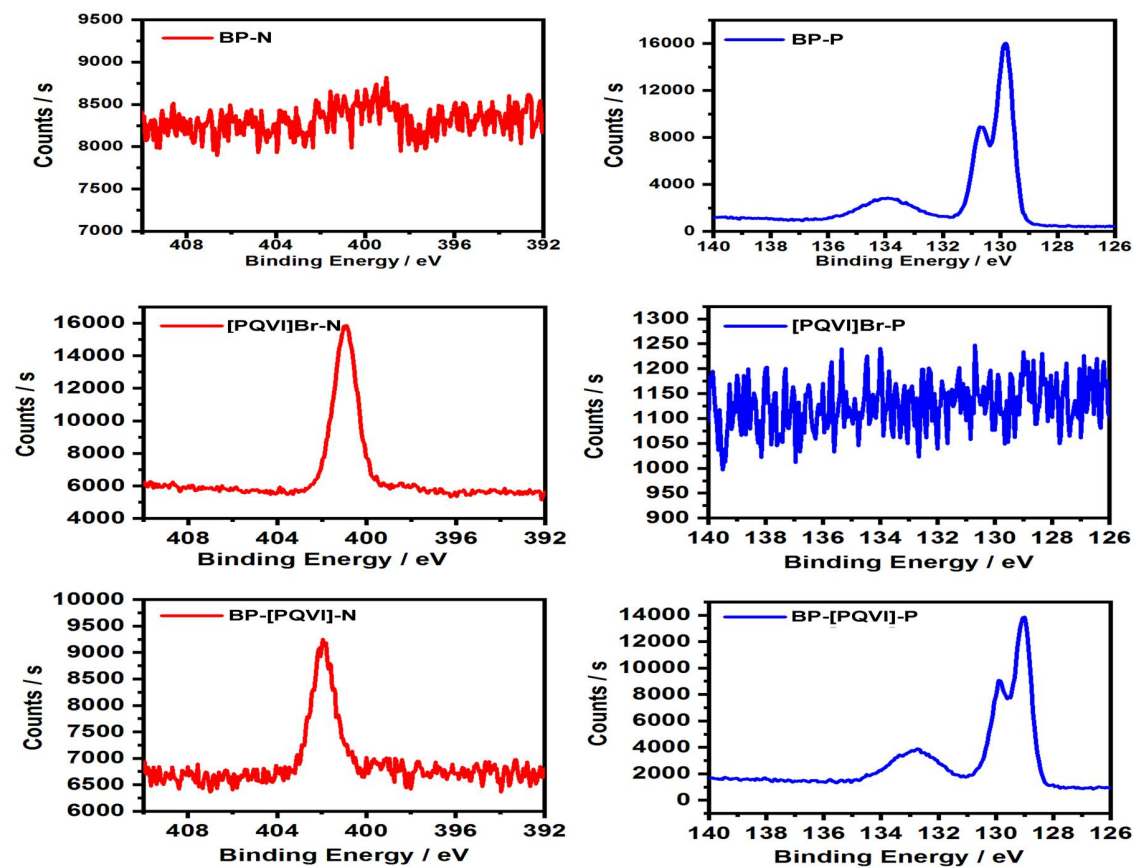

Figure S4. High-resolution XPS spectrum of [PQVI]Br and BP-PQVI. 


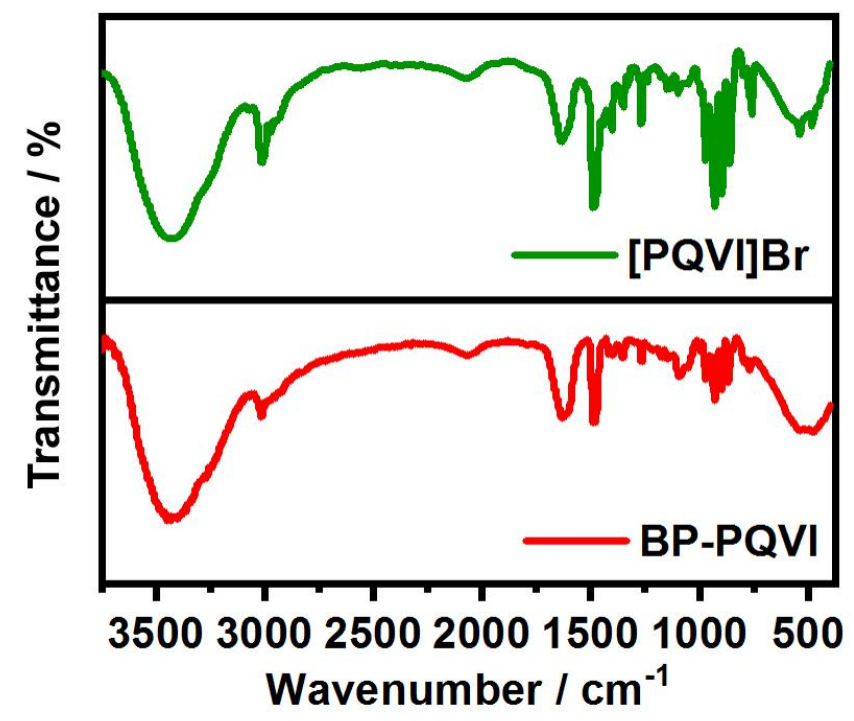

Figure S5. FT-IR spectra of [PQVI]Br and BP-PQVI. 


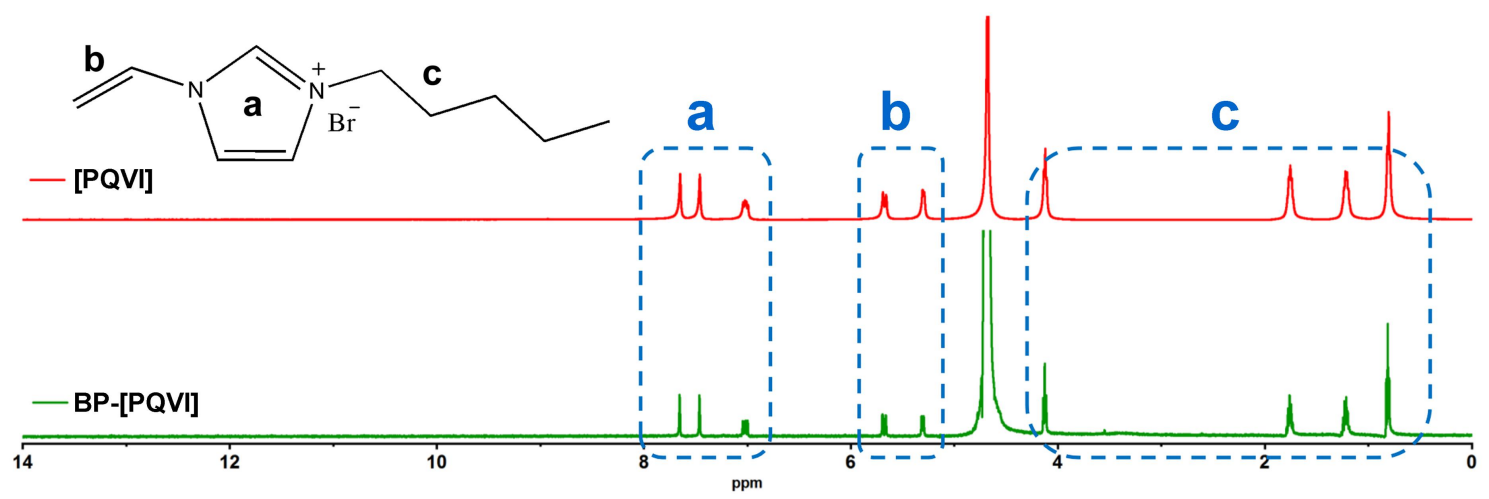

Figure S6. ${ }^{1} \mathrm{H}$ NMR spectra of PQVI and BP-PQVI, which a, b, and c corresponded to the characteristic peaks of the imidazole, vinyl, and alkane chains. 

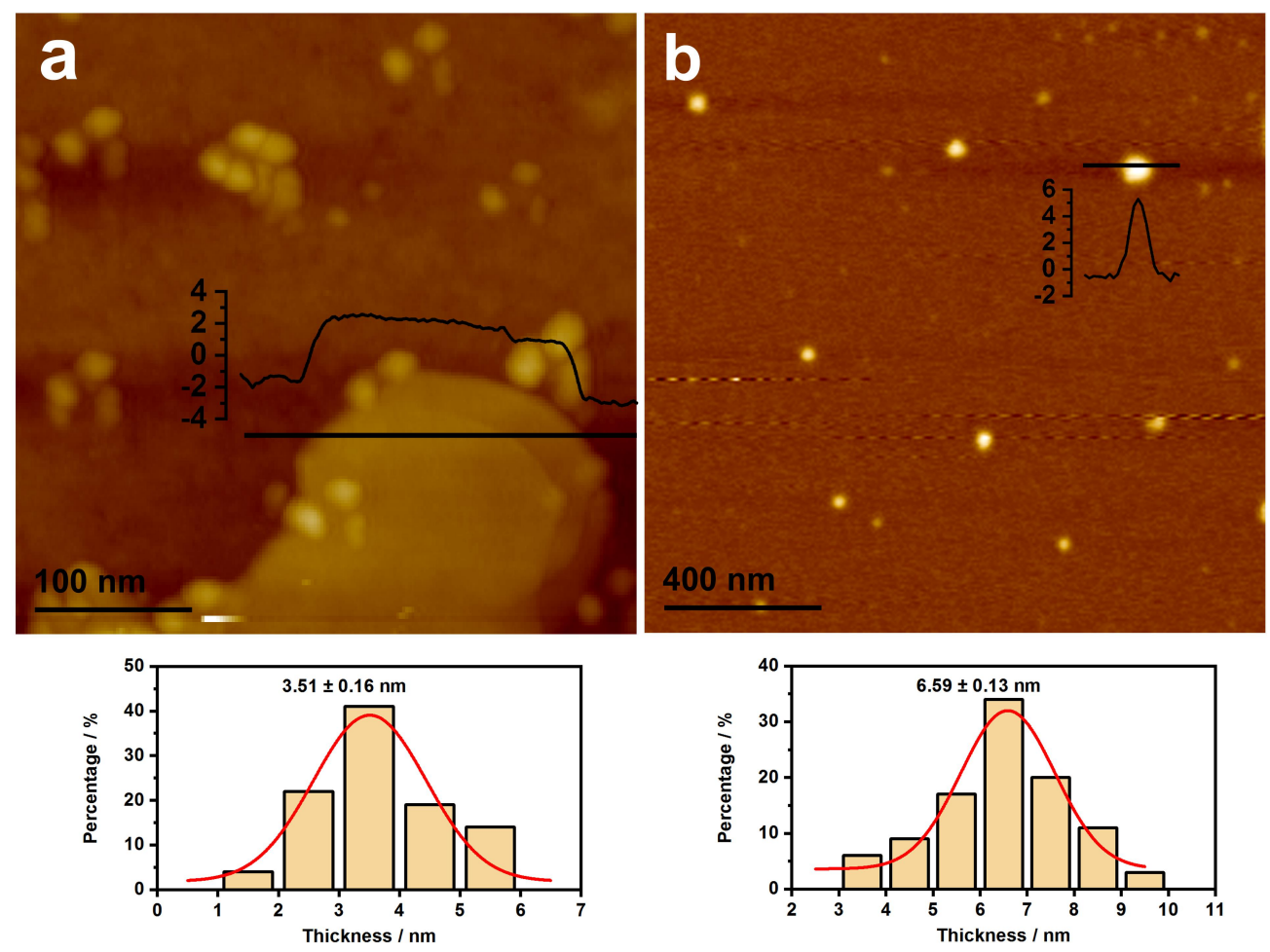

Figure S7. The distribution of thickness of BP obtained at a centrifugal speed of a) $15,000 \mathrm{rpm}$ and b) $1000 \mathrm{rpm}$. 

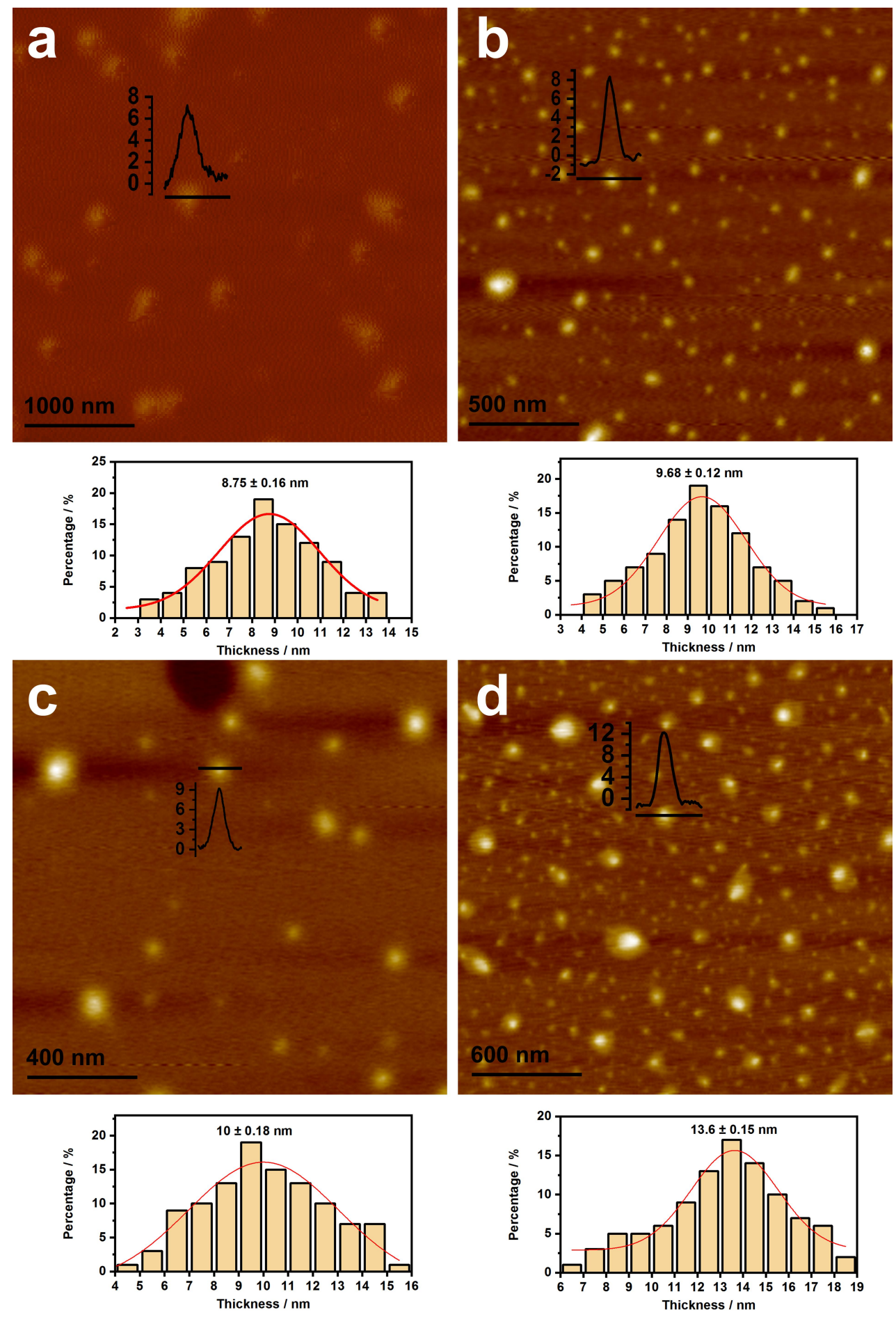

Figure S8. The distribution of thickness of BP-p[PQVI] obtained at a centrifugal speed of a) Assembly of 15,000 BP and p[PQVI] polymerized for 12 hours, b) Assembly of 15,000 BP and p[PQVI] polymerized for 24 hours, c) Assembly of $1000 \mathrm{BP}$ and $\mathrm{p}[\mathrm{PQVI}]$ 
polymerized for 12 hours and d) Assembly of $1000 \mathrm{BP}$ and $\mathrm{p}[\mathrm{PQVI}]$ polymerized for 24 hours. 

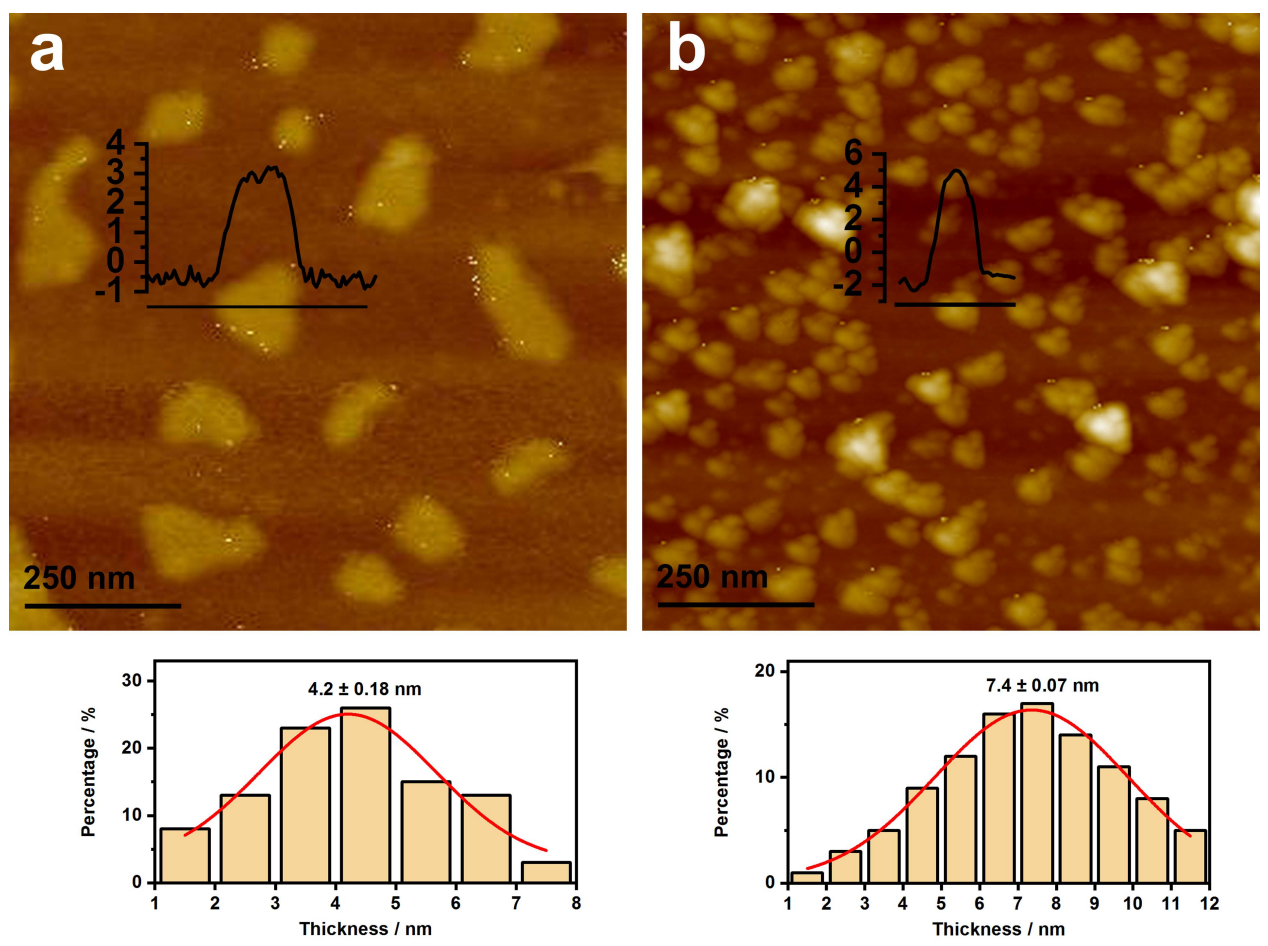

Figure S9. The distribution of thickness of BP-PQVI obtained at a centrifugal speed of a) Assembly of 15,000 BP and PQVI and b) Assembly of 1000 BP and PQVI. 

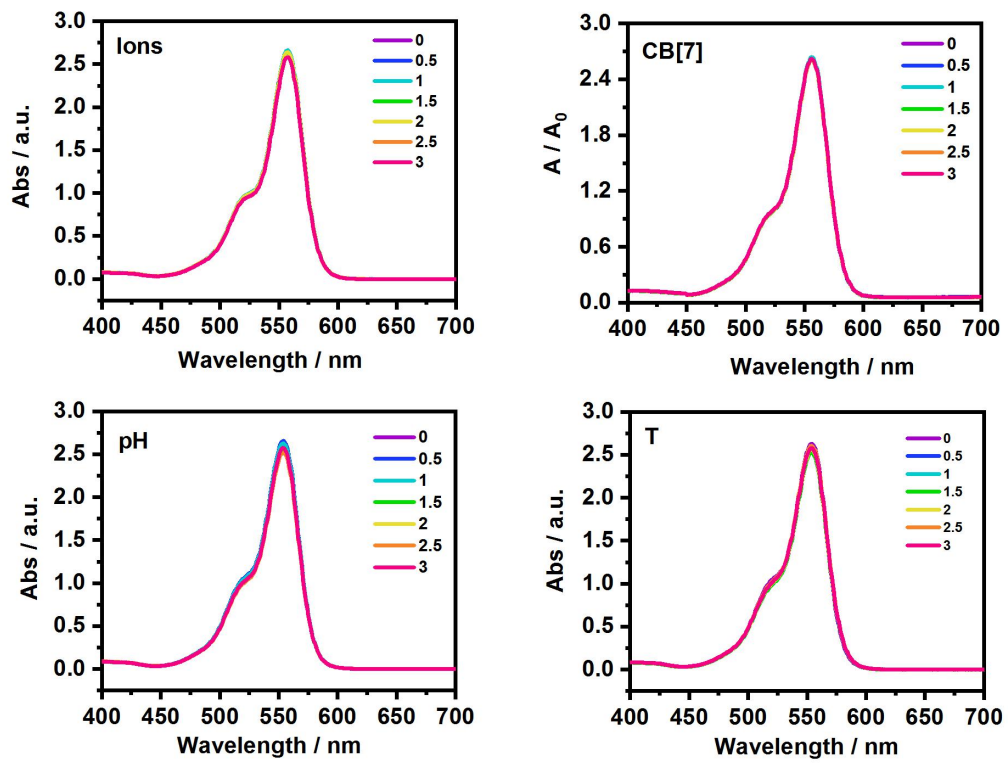

Figure S10. UV/Vis spectra of brilliant green (BG) in the presence of four external stimuli. 

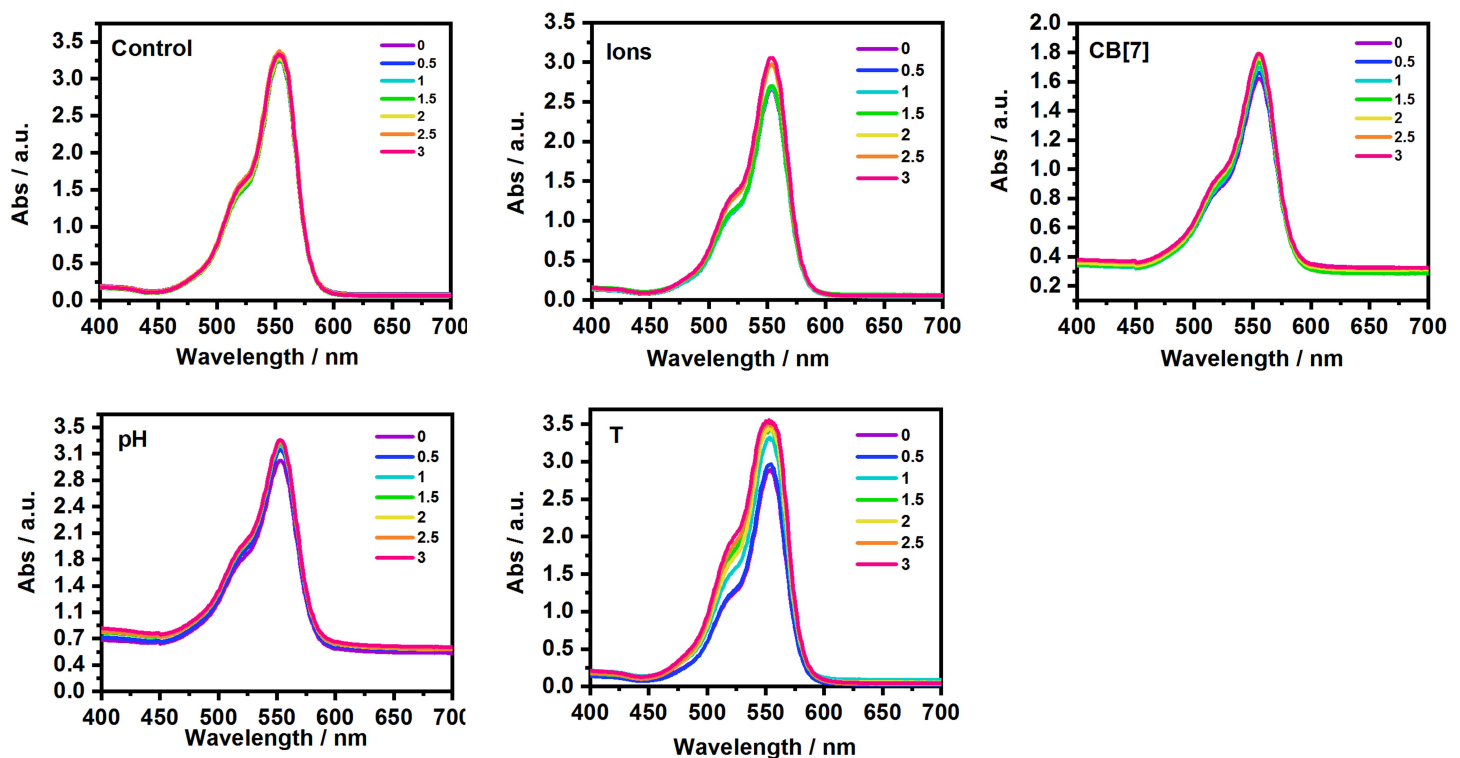

Figure S11. UV/Vis spectra of BP-BG supernate in the presence of four external stimuli. 

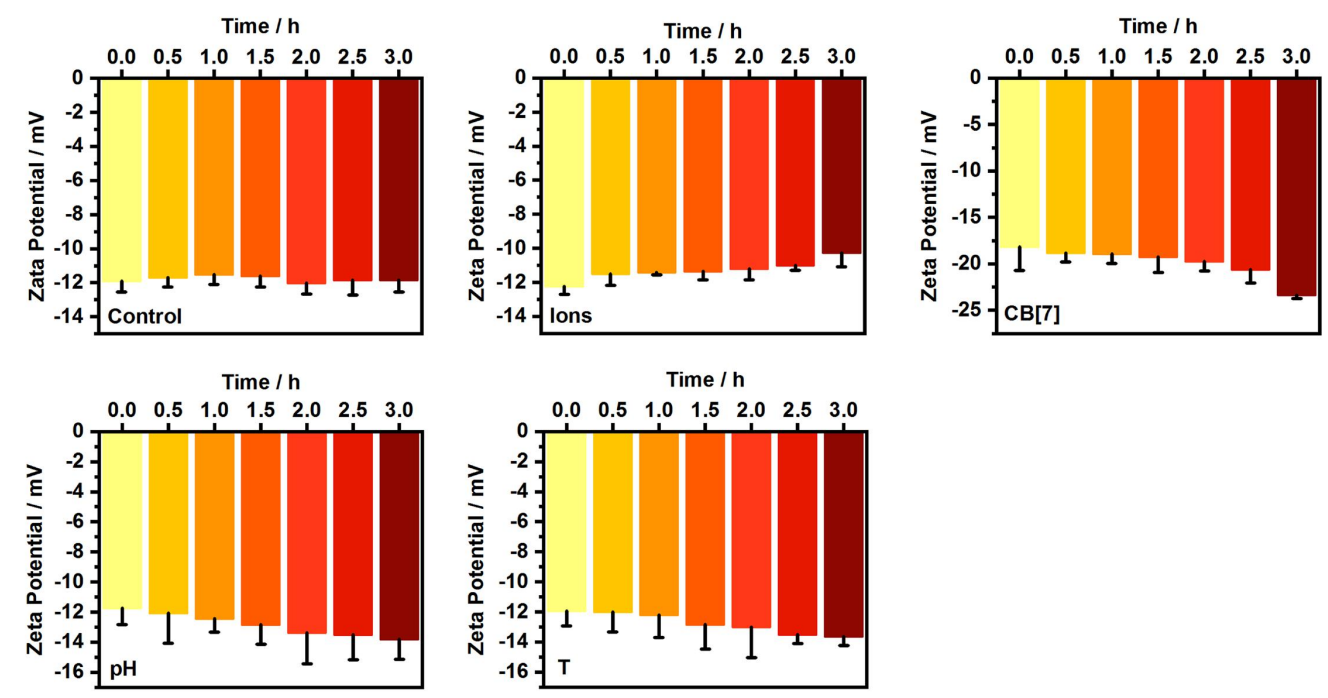

Figure S12. Zeta potential of BP-BG precipitate in the presence of four external stimuli. 


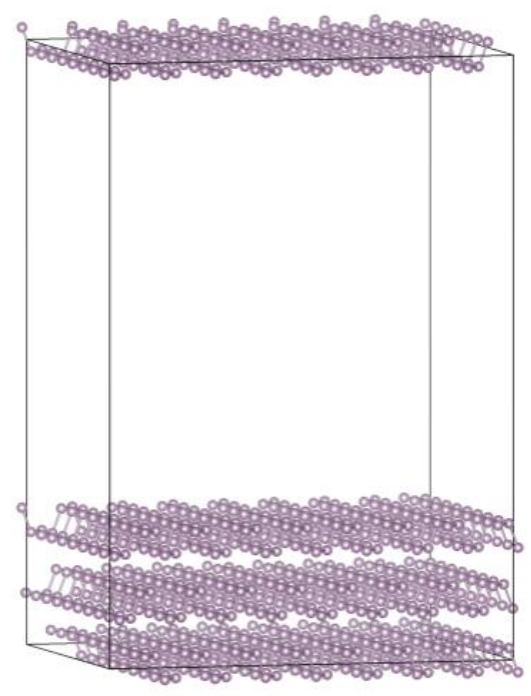

Figure S13. Crystal structure and adsorption model of BP. 


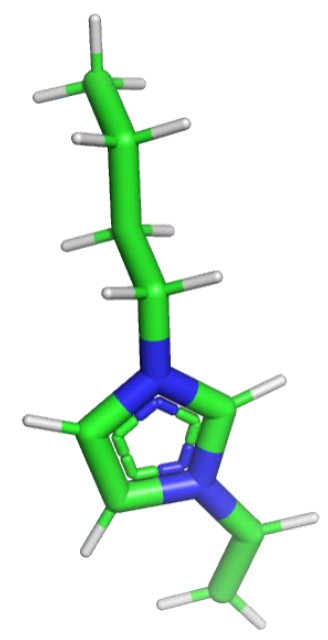

Figure S14. Molecular structure diagram of PQVI. 


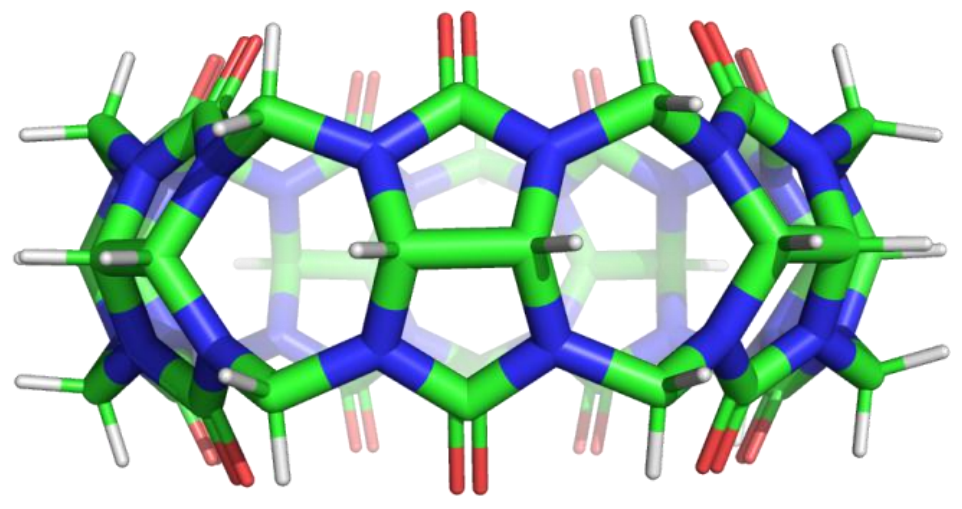

Figure S15. Molecular structure diagram of CB[7]. 


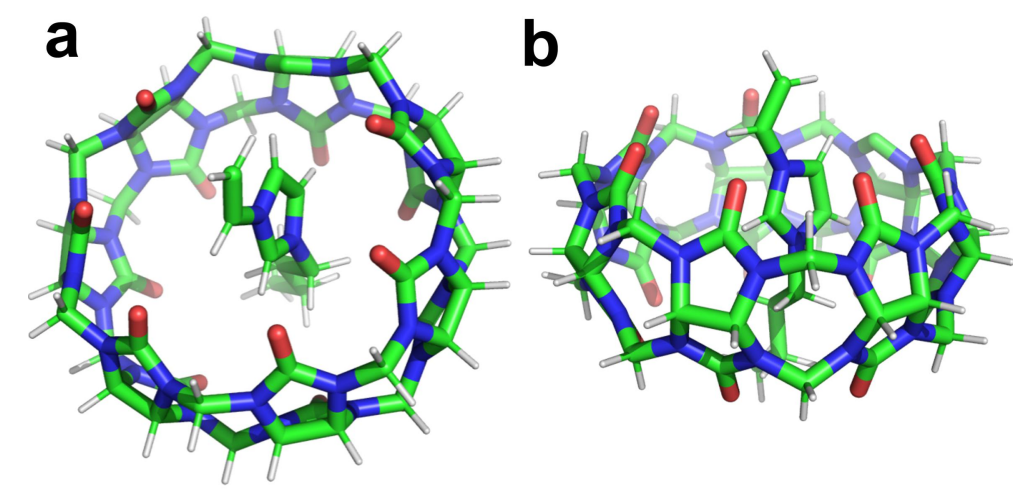

Figure S16. a) Top view and b) side view of the schematic diagram of the coating action of $\mathrm{CB}[7]$ molecule on PQVI. 

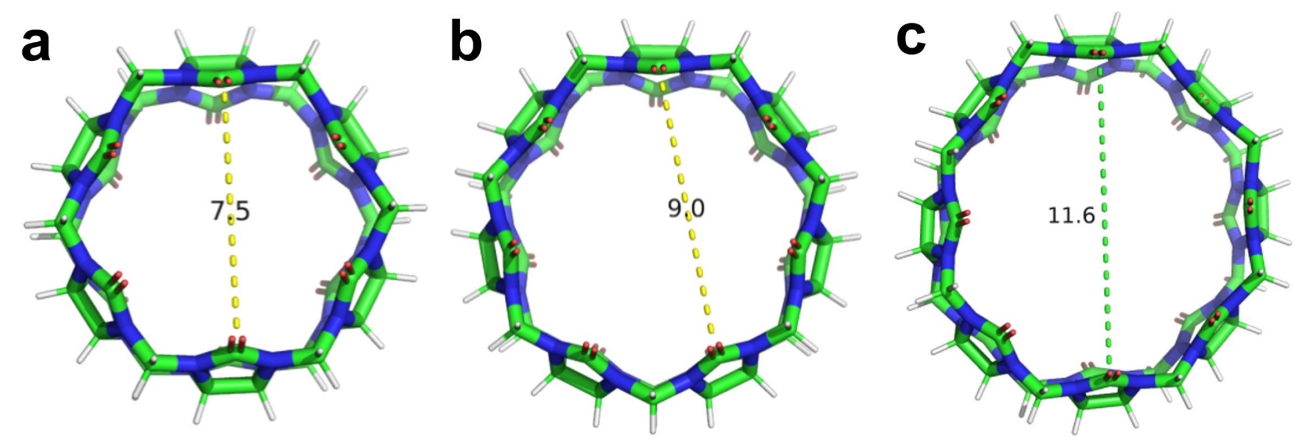

Figure S17. a) Schematic diagram of pore size of a) $\mathrm{CB}[6]$, b) $\mathrm{CB}[7]$, and c) $\mathrm{CB}[8]$. 


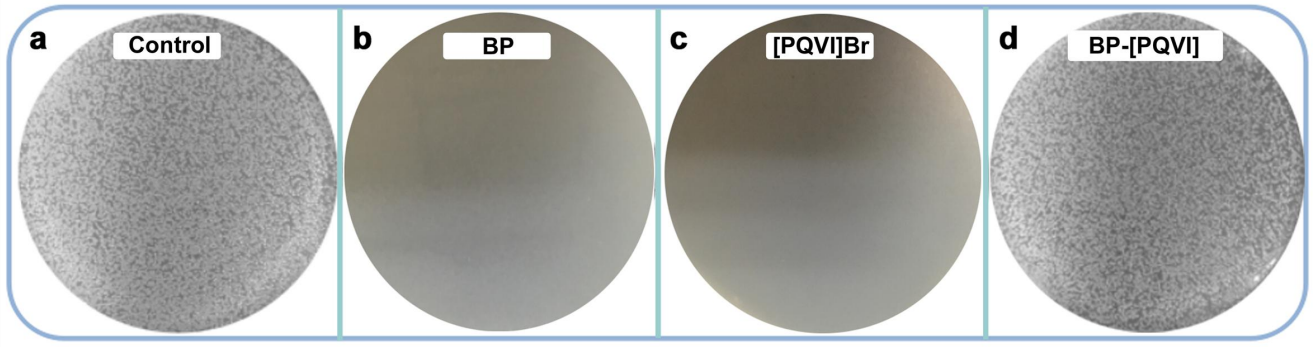

Figure S18. Digital images of $E$. coli on agar-LB plates after exposure to a) $\mathrm{NaCl}$ solution, b) BP, c) [PQVI]Br, and d) BP-PQVI, respectively. 


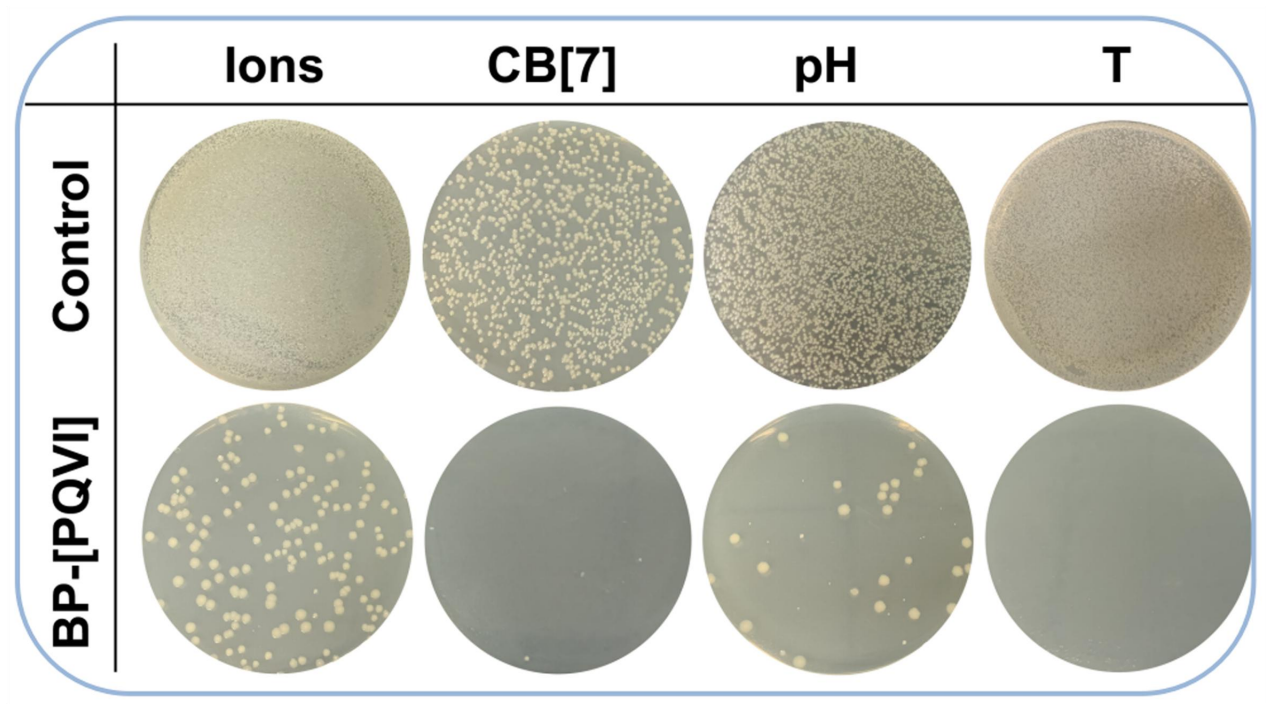

Figure S19. Digital images of $E$. coli on agar-LB plates after exposure to BP-PQVI in the presence of four external stimuli. 


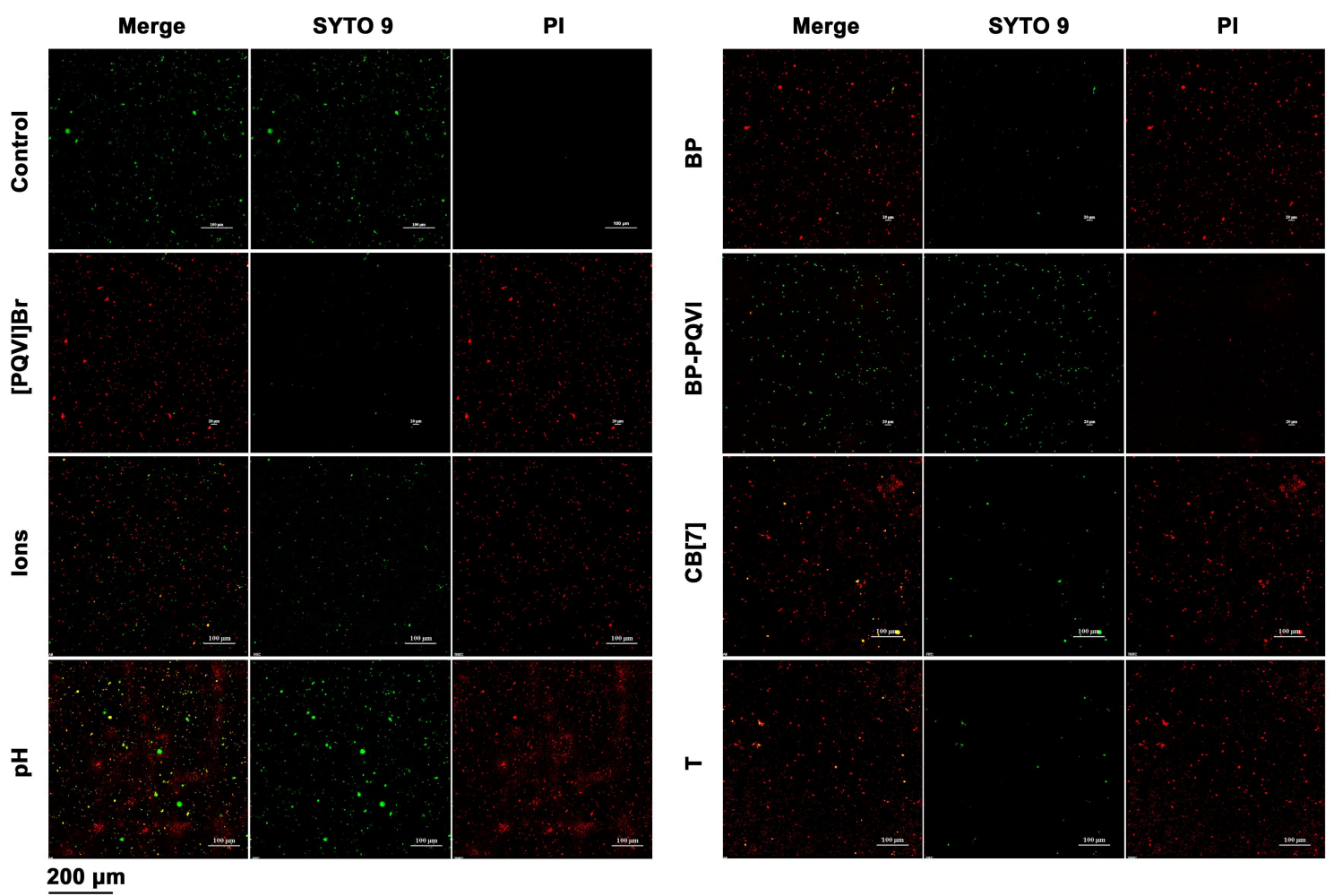

Figure S20. Live/dead staining assay of E. coli after treatment with BP, [PQVI]Br, BPPQVI and in the presence of four external stimuli. 